\title{
A Multifunctional Chemical Agent as an Attenuator of Amyloid Burden and Neuroinflammation in Alzheimer's Disease
}

Hong-Jun Cho, ${ }^{1}$ Anuj K. Sharma, ${ }^{4}$ Ying Zhang, ${ }^{3,5}$ Michael L. Gross, ${ }^{3}$ and Liviu M. Mirica ${ }^{1,2, *}$

${ }^{1}$ Department of Chemistry, University of Illinois at Urbana-Champaign, 600 S. Mathews Avenue, Urbana, Illinois 61801, United States

${ }^{2}$ Hope Center for Neurological Disorders, Washington University School of Medicine, St. Louis, MO 63110, United States

${ }^{3}$ Department of Chemistry, Washington University, One Brookings Drive, St. Louis, MO 63130, United States

${ }^{4}$ Department of Chemistry, Central University of Rajasthan, NH-8, Bandarsindri, Ajmer, Rajasthan 305817, India

${ }^{5}$ Current address: Pfizer Inc, 1 Burtt Rd, Andover, MA 01810, United States

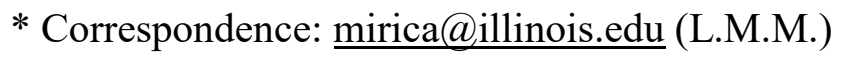




\section{Table of Contents}

1. General methods $\quad$ S3

2. $\mathrm{A} \beta$ peptide preparation $\quad \mathrm{S} 3$

3. Trolox equivalent antioxidant capacity (TEAC) assays S4

4. Coumarin-3-carboxylic acid (CCA) assays $\quad$ S4

5. Pulsed hydrogen-deuterium exchange (HDX) assays S5

6. Pulsed HDX data analysis and processing S6

7. Cell viability studies $\quad$ S6

8. Two-photon in vivo imaging studies $\quad$ S8

9. Fluorescence microscopy images of the brain sections S10

10. Quantification of amyloid plaques S11-S14

11. Fluorescence images of the brain sections immunostained with S15 AF594-Iba1 antibody

12. Fluorescence images of the brain sections immunostained with S16 AF594-AT8 antibody

13. References 


\section{General methods}

Unless otherwise noted, all chemical reagents and solvents were purchased from commercial suppliers and used without further purification. The Neuro-2a (N2A) mouse neuroblastoma cell line was purchased from the American Type Culture Collection (ATCC) and cultivated in Dulbecco's Modified Eagle Medium (DMEM) supplemented with 10\% FBS and 1\% antibiotic (penicillin-streptomycin) in a humidified $5 \% \mathrm{CO}_{2}$ incubator at $37{ }^{\circ} \mathrm{C} .5 \times \mathrm{FAD}$ transgenic mice (Tg6799 line) overexpressing mutant human APP (695) with the Swedish (K670N, M671L), Florida (I716V), and London (V717I) were purchased from Jackson Laboratories (Bar Harbor, ME, USA). Monoclonal anti-A $\beta$ antibody HJ3.4 was obtained from Prof. David Holtzman (Department of Neurology, Washington University School of Medicine). ${ }^{1}$ Iba1 polyclonal antibody (Iba1) and phospho-Tau (Ser202, Thr205) monoclonal antibody (AT8) were purchased from ThermoFisher Scientific. The antibodies were directly labeled with Alexa Fluor ${ }^{\mathrm{TM}} 594$ using an Antibody Labeling Kit (ThermoFisher Scientific), in accordance with the protocol provided by the manufacturer.

\section{A $\beta$ peptide preparation}

All $A \beta_{42}$ monomeric films were prepared following a literature protocol. ${ }^{2}$ Briefly, the $A \beta_{42}$ peptide (Keck Biotechnology Resource Laboratory, Yale University or rPeptide) was dissolved in hexafluoroisopropyl alcohol (HFIP, $1 \mathrm{mM}$ ) and incubated for $1 \mathrm{~h}$ at room temperature. The clear solution was transferred into low-binding Eppendorf tubes and allowed to evaporate overnight. The aliquots were dried by vacuum centrifugation for $10 \mathrm{~min}$, and the resulting films of monomeric $\mathrm{A} \beta_{42}$ were stored at $-80^{\circ} \mathrm{C}$. 


\section{Trolox equivalent antioxidant capacity (TEAC) assays}

The antioxidant activity of L1 was evaluated by the TEAC assay as described in the literature. ${ }^{3-4}$ 2,2'-Azino-bis(3-ethylbenzothiazoline-6-sulphonic acid) (ABTS, $16.46 \mathrm{mg}, 30 \mu \mathrm{mol}$ ) and potassium persulfate $(3.24 \mathrm{mg}, 12 \mu \mathrm{mol})$ were dissolved in $4 \mathrm{~mL}$ of water and the mixture was shaken overnight in the dark at room temperature to generate the $\mathrm{ABTS}^{-+}$radical cation. The ABTS $^{++}$solution $(30 \mu \mathrm{L})$ was diluted with methanol to a final volume of $300 \mu \mathrm{L}$ to give an absorbance of 0.711 at $470 \mathrm{~nm}$. Stock solutions $(1 \mathrm{mM})$ of Trolox, glutathione, and L1 were prepared in methanol. Each solution $(30 \mu \mathrm{L}$, for a final concentration of $100 \mu \mathrm{M})$ was added into 96-well plate and diluted with methanol to make a final volume of $270 \mu \mathrm{L}$. After adding $30 \mu \mathrm{L}$ of the $\mathrm{ABTS}^{\circ+}$ solution into each well, the absorbance at $470 \mathrm{~nm}$ was monitored at selected time points $(1,3,6$, and $15 \mathrm{~min})$. The assays were performed with various concentrations of the compounds $(25,50,75$ and $100 \mu \mathrm{M})$ and carried out in triplicate. The percent inhibition was calculated based on the absorbance $\left(\%\right.$ inhibition $\left.=100 \times\left(\mathrm{A}_{0}-\mathrm{A}\right) / \mathrm{A}_{0}\right)$, and then was plotted as a function of the concentration of the compound. The TEAC values of each compound were obtained after normalization of the slope by that of Trolox.

\section{Coumarin-3-carboxylic acid (CCA) assays}

The CCA was used to measure the production of hydroxyl radical induced by $\mathrm{Cu}$ ions. ${ }^{4}$ The stock solutions $(10 \mathrm{mM})$ of CCA, $\mathrm{CuSO}_{4}$, ascorbic acid, and $\mathrm{L} 1$ were prepared in water. Each compound was added into 96-well plate and diluted with PBS (1×, pH 7.4) to make a solution with the following concentrations: $\mathrm{CCA}[100 \mu \mathrm{M}], \mathrm{CuSO}_{4}[40 \mu \mathrm{M}]$, ascorbic acid $[400 \mu \mathrm{M}]$, and $\mathrm{L} 1[0-80$ $\mu \mathrm{M}$ ] (final volume: $200 \mu \mathrm{L}$ ). The fluorescence from 7-hydroxycoumarin-3-carboxylic acid was 
then monitored upon excitation at $395 \mathrm{~nm}$ and emission at $450 \mathrm{~nm}$ for $1 \mathrm{~h}$ using a SpectraMax M2e plate reader (Molecular Devices, USA).

\section{Pulsed hydrogen-deuterium exchange (HDX) assays}

A monomeric film of $A \beta_{42}$ was dissolved in anhydrous DMSO $(1 \mathrm{mM})$, and $A \beta_{42}$ aggregation was induced by dilution with PBS buffer $(1 \times, \mathrm{pH} 7.4)$ at $37^{\circ} \mathrm{C}$ in a $1: 19(\mathrm{v} / \mathrm{v})$ ratio, in the presence of $\mathrm{CuCl}_{2}$ and $\mathrm{L} 1$ at the same concentration as that of $\mathrm{A} \beta_{42}$ (final concentration of $\mathrm{A} \beta_{42}, \mathrm{Cu}^{2+}$, and $\mathrm{L} 1$ : $50 \mu \mathrm{M})$. The final solution was horizontally agitated at $150 \mathrm{rpm}$ for $42 \mathrm{~h}$. The samples were collected at selected time points (13 times) for hydrogen-deuterium exchange (HDX) analysis. Soluble $A \beta_{42}$ aggregates were enriched by centrifugation $\left(16,000 \times \mathrm{g}, 5 \mathrm{~min}\right.$ at $\left.4{ }^{\circ} \mathrm{C}\right)$, and the centrifuged samples were carefully divided into two equal-volume parts (10 $\mu \mathrm{L}$ each). The lower half of the sample was submitted to MS analysis to generate the plots shown in figures, using the method reported previously. ${ }^{5}$

The HDX experiments were carried out by mixing the sample solution and $\mathrm{D}_{2} \mathrm{O}$ buffer (pD 7.4 PBS in $\left.\mathrm{D}_{2} \mathrm{O}\right)$ in a $1: 1(\mathrm{v} / \mathrm{v})$ ratio for 1 min at $0{ }^{\circ} \mathrm{C}$. The $\mathrm{HDX}$ reaction was quenched by adding 30 $\mu \mathrm{L}$ of $3 \mathrm{M}$ urea with $1 \%$ TFA into the solution to make a $\mathrm{pH} 2.5$ solution. The quenched solution was passed through a custom-packed pepsin column at $200 \mu \mathrm{L} / \mathrm{min}$ for digestion, and the peptic peptides were captured on a C8 trap column $(2 \mathrm{~mm} \times 1 \mathrm{~cm}$, Agilent Inc., Santa Clara, CA $)$ and desalted (the total time for digestion and desalting was $3 \mathrm{~min}$ ). The peptides were then separated with a linear gradient of $4-40 \%$ of $\mathrm{CH}_{3} \mathrm{CN}$ in $0.1 \%$ formic acid (v/v) over $5 \mathrm{~min}$. Both the peptide digestion and separation were performed at $0{ }^{\circ} \mathrm{C}$ to minimize back-exchange. The eluted peptides were analyzed by a MaXis quadrupole time-of-flight (Bruker Daltonics Inc., Germany) in the 
positive-ion electrospray ionization mode. All analyses were done in triplicate.

\section{Pulsed HDX data analysis and processing}

Peptic peptides identification was performed as described previously. The centroid mass of the peptides was converted by MagTran v1.03. The percent protection was calculated by using Eq. 1:

$$
\% \text { protection }=100 \%-\% \mathrm{D}=\left(1-\frac{m_{H D X}-m_{\text {control }}}{(N-2) \times 0.5}\right) \times 100 \% \quad \text { Eq } 1
$$

where $\mathrm{m}_{\mathrm{HDX}}$ is the centroid mass of the deuterated peptides, $\mathrm{m}_{\text {control }}$ was the centroid mass of nondeuterated peptides, $(\mathrm{N}-2)$ is the number of exchangeable amide hydrogens, and 0.5 is the final $\mathrm{D}_{2} \mathrm{O}$ content of the buffer system. The experimentally determined time-dependent data (mass shift versus incubation time) were characterized phenomenologically by following the recommendation of Finke and coworkers to use the simplest model consistent with the data, ${ }^{6}$ and all kinetic curves were fitted with a modified F-W model. ${ }^{5}$

\section{Cell viability studies}

The N2A cells were seeded $\left(2.5 \times 10^{4}\right.$ cells/well $)$ onto 96 -well plates with DMEM/10\% FBS and incubated for $24 \mathrm{~h}$. The media was replaced with serum-free medium containing N2 supplement. After $1 \mathrm{~h}$, the $\mathrm{A} \beta_{42}$ peptide $[20 \mu \mathrm{M}], \mathrm{CuCl}_{2}[20 \mu \mathrm{M}]$, and $\left.\mathrm{L} 1[2 \mu \mathrm{M}]\right)$ were added into each well in different conditions $\left(A \beta_{42}\right.$ only, $L 1$ only, $A \beta_{42}$ oligomers only, $\left.A \beta_{42}+C u, A \beta_{42}+C u+L 1\right)$, followed by incubation at $37^{\circ} \mathrm{C}$. The final volume in each well was $100 \mu \mathrm{L}$, with up to $1 \%$ DMSO. After $40 \mathrm{~h}$, each well was treated with $10 \mu \mathrm{L}$ of Alamar blue reagent and the cells were incubated 
for $1.5 \mathrm{~h}$. Absorbance was measured at $570 \mathrm{~nm}$ (control $\mathrm{OD}=600 \mathrm{~nm}$ ) using a SpectraMax M2e plate reader (Molecular Devices, USA).

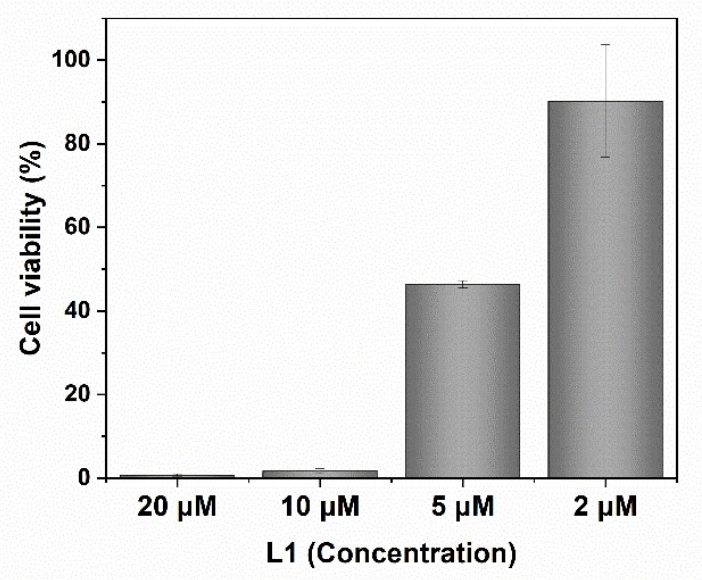

Figure S1. Cell viability of N2A cells (normalized to a $1 \%$ DMSO control) after $40 \mathrm{~h}$ treatment with L1, as assessed by the Alamar Blue assay. Conditions: [L1] $=2-20 \mu \mathrm{M}$. 


\section{Two-photon in vivo imaging studies}

The handling and imaging of mice were performed in accordance with institutional guidelines. 8month old $5 \mathrm{xFAD}$ and WT mice were placed in a stereotaxic frame with ear bars under $2 \%$ isoflurane, and the skin and periosteum were removed to expose the skull. Compound L1 (500 $\mu \mathrm{M}$, $1 \%$ DMSO in PBS) was directly administrated into the brain via intracranial injection at three points at the edge of the cranial window area. The injection rate was $0.2 \mu \mathrm{L} / \mathrm{min}$ for $10 \mathrm{~min}$ at each point. After intracranial injections, thinned-skull cranial windows were prepared as previously described. ${ }^{7}$ The skull was thinned with a high-speed drill and scraped with a microsurgical blade until it was transparent and flexible. One cranial window $(5 \times 5 \mathrm{~mm})$ was prepared over the barrel cortex of a hemisphere of the brain. Then, the mice were mounted on a custom-built stereotaxic apparatus for two-photon imaging and a small ring of molten bone wax was applied to the skull surrounding the perimeter of the window to create a water immersion chamber. The cranial window was placed under the objective lens on a two-photon microscope (Zeiss LSM510 META NLO) with tunable Coherent Chameleon Ultra I laser. Prior to two photon imaging, mice were intravenously injected with Texas Red-dextran conjugate (70 kDa molecular weight, $200 \mu \mathrm{L}, 25$ $\mathrm{mg} / \mathrm{kg}$ dissolved in PBS ( $\mathrm{pH}$ 7.4)) via cannulated jugular veins to see blood vessels. The fluorescence of L1 and Texas Red were monitored with 435-485 nm and 565-615 nm emission filters under excitation of $770 \mathrm{~nm}$ and $543 \mathrm{~nm}$, respectively. 

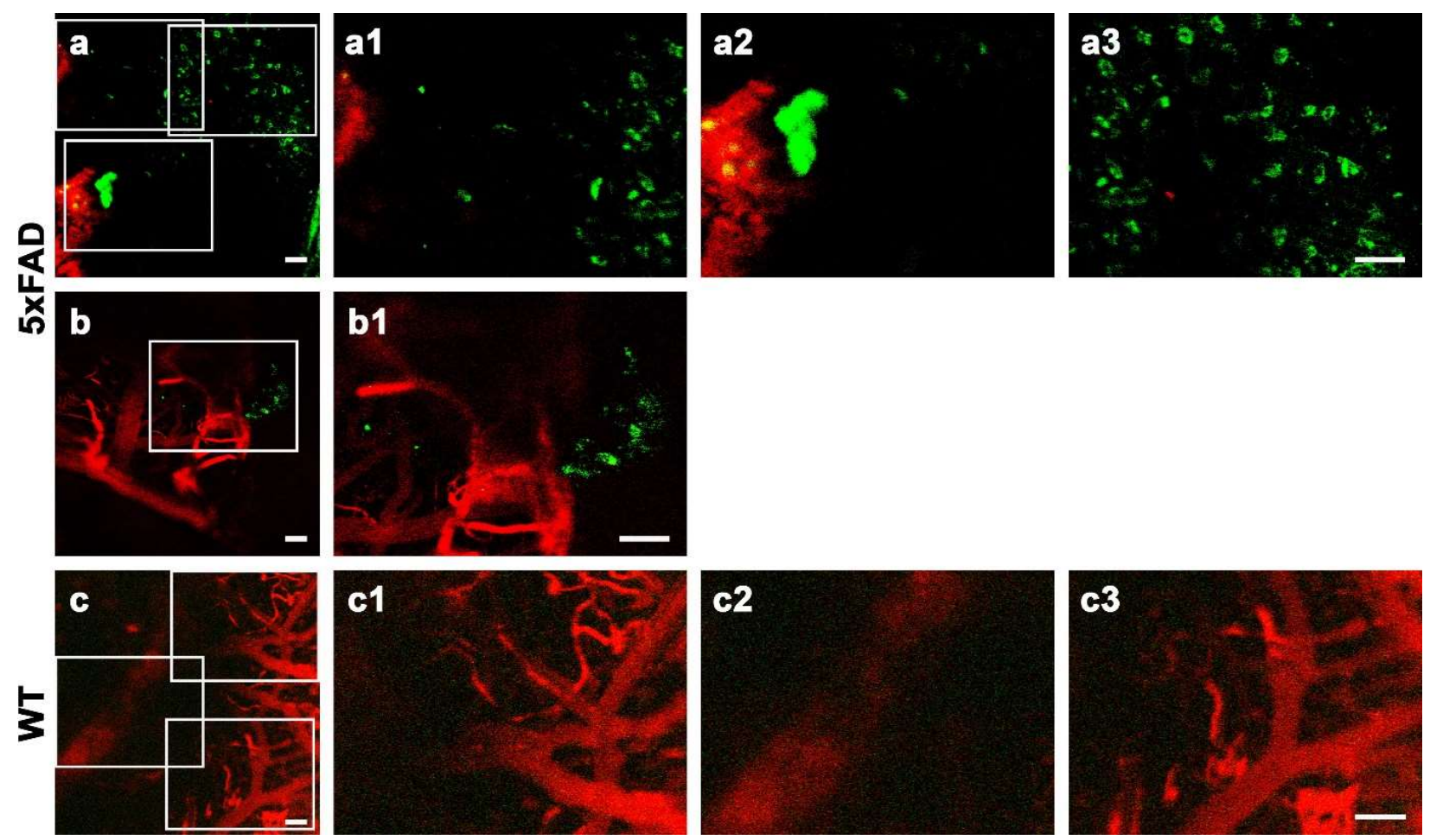

Figure S2. In vivo two-photon fluorescence brain images of AD (a and b) or WT (c) mice after intracranial injection of L1. The fluorescence from L1 was monitored at 435-485 nm under excitation of two-phone excitation at $770 \mathrm{~nm}$. For blood vessels staining, dextran-Texas Red (70 $\mathrm{kDa}$ ) was intravenously injected prior to two-photon imaging. The fluorescence from Texas Red was monitored at 565-615 nm under excitation of excitation at $543 \mathrm{~nm}$. The regions in each image with white rectangle are magnified. Scale bar: $50 \mu \mathrm{m}$. 
Bright field

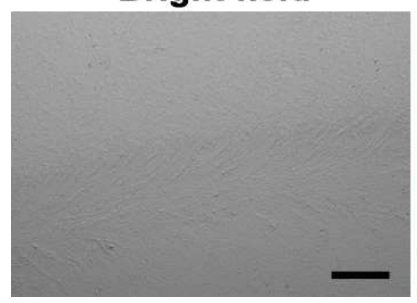

AF594-HJ3.4

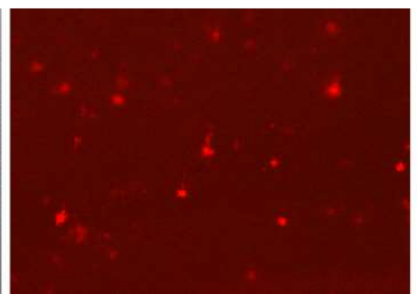

L1

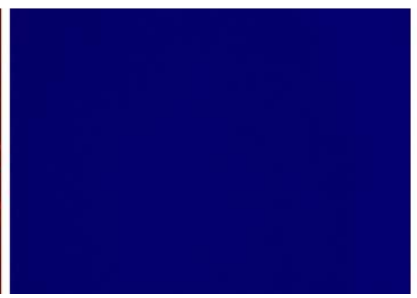

Merged

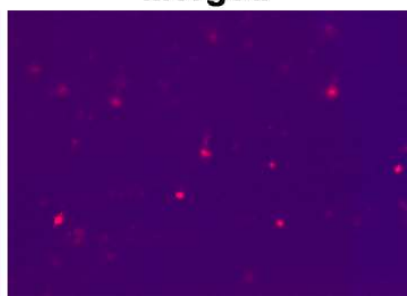

Figure S3. Fluorescence microscopy images of brain sections from 5xFAD mice treated with vehicle for 30 days. The brain sections were immunostained with the AF594-HJ3.4 antibody. The fluorescence signals from AF594-HJ3.4 antibody and lack of L1 emission were monitored at 600$660 \mathrm{~nm}$ and $435-485 \mathrm{~nm}$ under excitation at 340-380 $\mathrm{nm}$ and 540-580 nm, respectively (AF594HJ3.4 antibody: red; L1: blue, showing no background emission; scale bar: $100 \mu \mathrm{m})$.
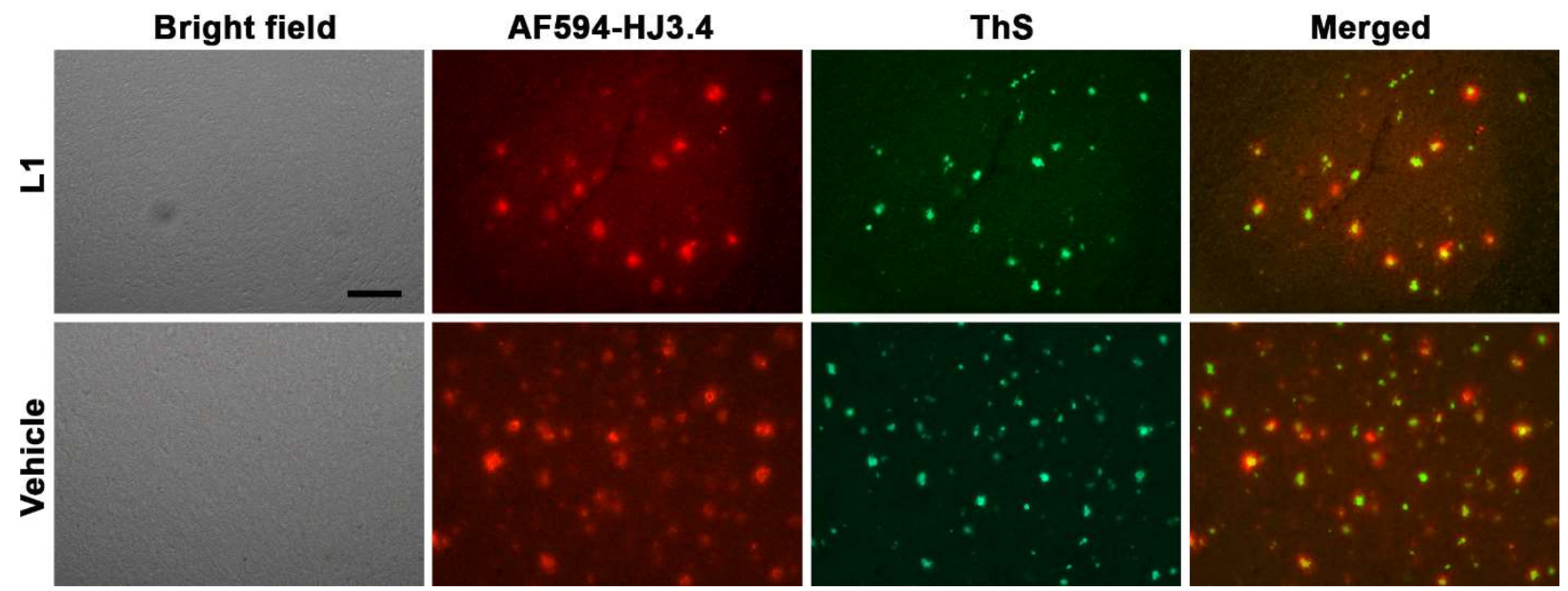

Figure S4. Fluorescence microscopy images of the brain sections from 5xFAD mouse treated with L1 or vehicle. The brain sections were co-stained with AF594-HJ3.4 antibody and ThS. The fluorescence signals from AF594-HJ3.4 antibody and ThS were monitored at 600-660 nm and 515-555 nm under excitation at 540-580 nm and 465-495 nm, respectively. Color: red, AF594HJ3.4 antibody; green, ThS. Scale bar: $100 \mu \mathrm{m}$. ThS was strongly colocalized with AF594-HJ3.4 in the amyloid plaques (Pearson's correlation coefficients of 0.52 and 0.59 for the L1 - and vehicletreated brains). 
(a)
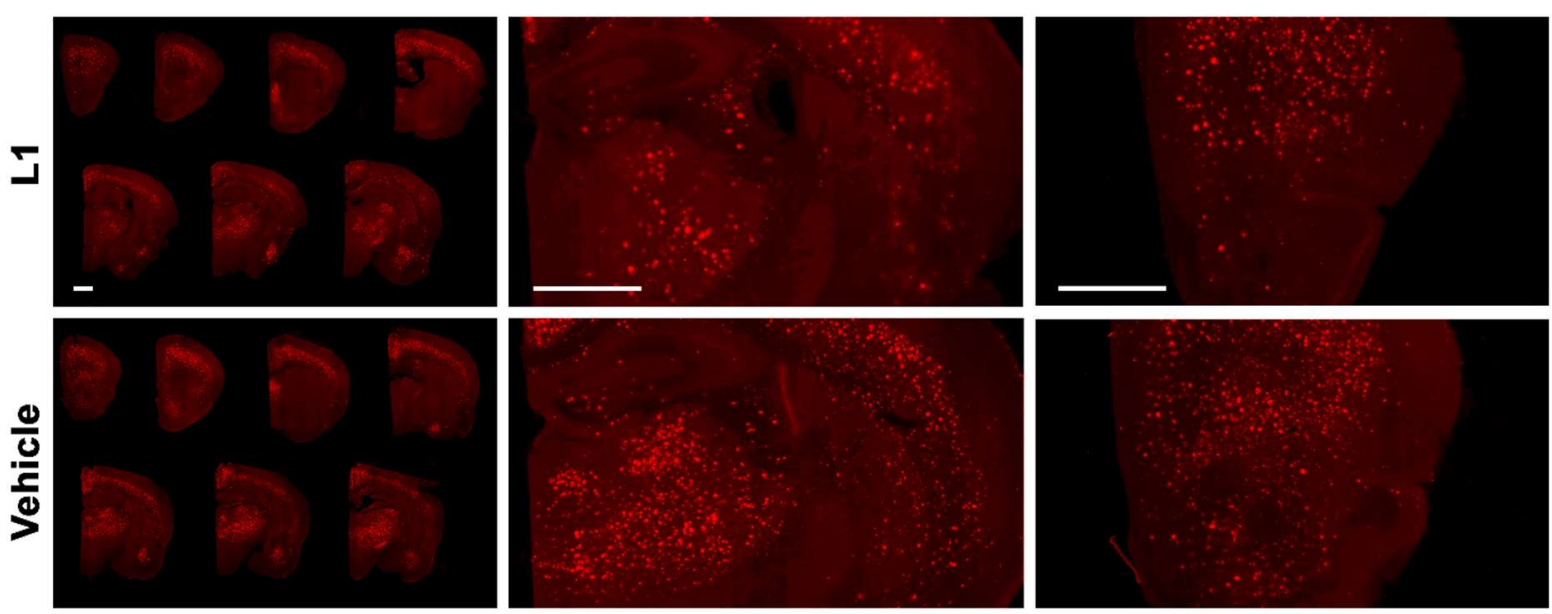

(b)

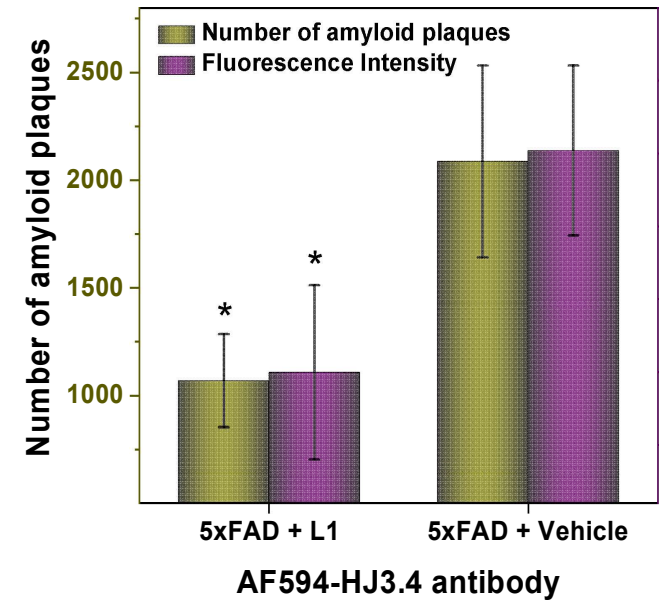

(c)

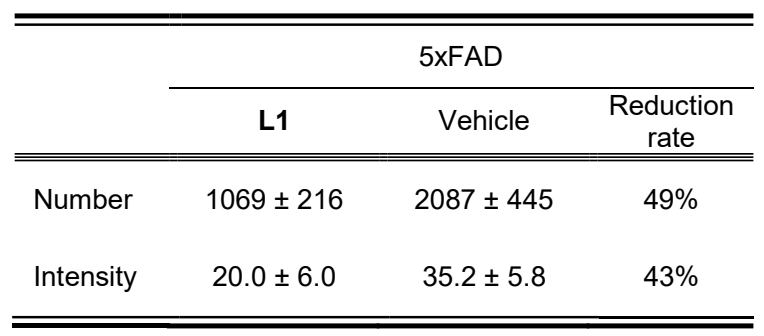

Figure S5. (a) Representative fluorescence microscopy images of the AF594-HJ3.4 antibodystained brain sections from 5xFAD mouse treated with L1 or vehicle. The fluorescence of AF594 was monitored with a standard TRITC filter set. Scale bar: $1 \mathrm{~mm}$. (b) The total number and fluorescence intensity of amyloid plaques in the AF594-HJ3.4 antibody-stained brain sections from $5 \mathrm{xFAD}$ mouse treated with $\mathrm{L} 1$ or vehicle. (c) When compared to vehicle-treated mice, the amyloid burden in the L1-treated mice was reduced by $49 \%$ and $43 \%$ based on the number of amyloid plaques and fluorescence intensity, respectively. The fluorescence intensity and number of amyloid plaques were obtained as the sum obtained from seven brain sections per mouse. Error bars are presented as standard deviation ( $\mathrm{n}=3$ mice), and the statistical analysis was evaluated according to one-way ANOVA $\left({ }^{*} \mathrm{p}<0.05\right)$. 
(a)
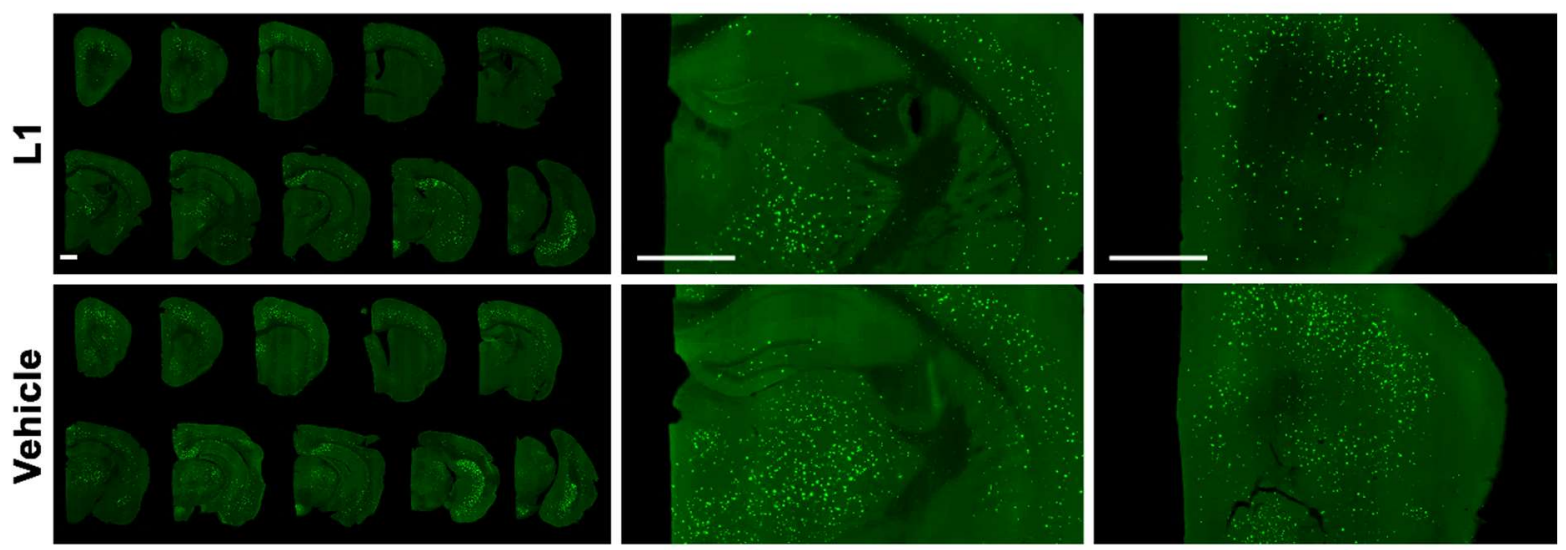

(b)

(c)
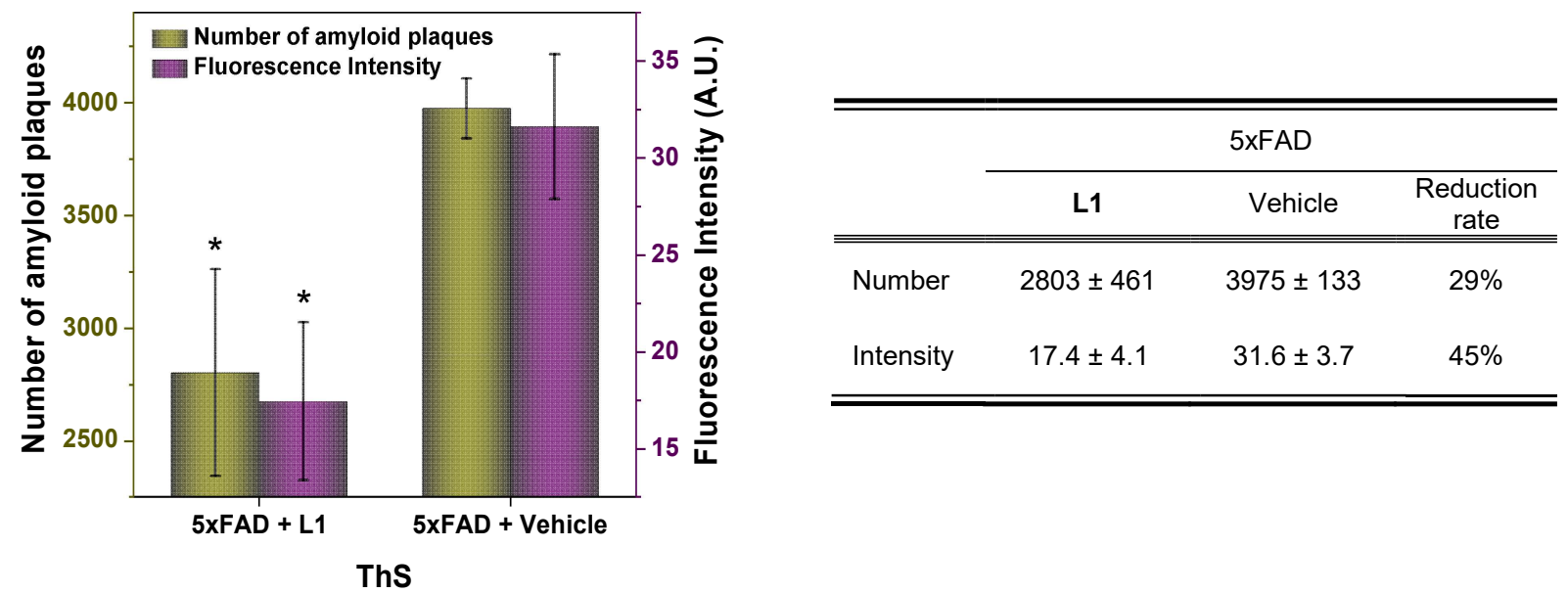

Figure S6. (a) Representative fluorescence microscopy images of the ThS-stained brain sections from $5 \mathrm{xFAD}$ mouse treated with $\mathrm{L} 1$ or vehicle. The fluorescence of ThS was monitored with a standard FITC filter set. The image in right lane is a magnified view. Scale bar: $1 \mathrm{~mm}$. (b) The total number and fluorescence intensity of amyloid plaques in the ThS-stained brain sections from 5xFAD mouse treated with L1 or vehicle. (c) When compared to vehicle-treated mice, the amyloid burden in the L1-treated mice were reduced by $29 \%$ and $45 \%$ based on the number of amyloid plaques and fluorescence intensity, respectively. The fluorescence intensity and number of amyloid plaques were obtained as the sum obtained from ten brain sections per mouse. Error bars are presented as standard deviation ( $\mathrm{n}=3$ mice), and the statistical analysis was evaluated according to one-way ANOVA $(* \mathrm{p}<0.05)$. 
(a)
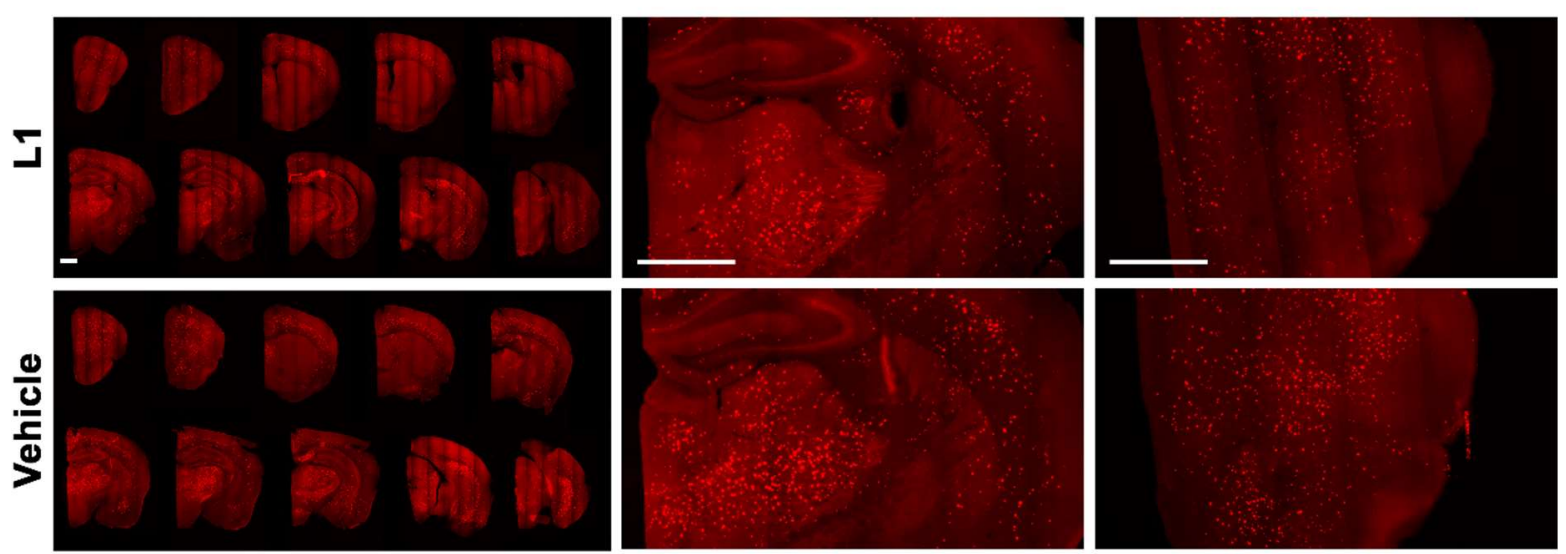

(b)

(c)
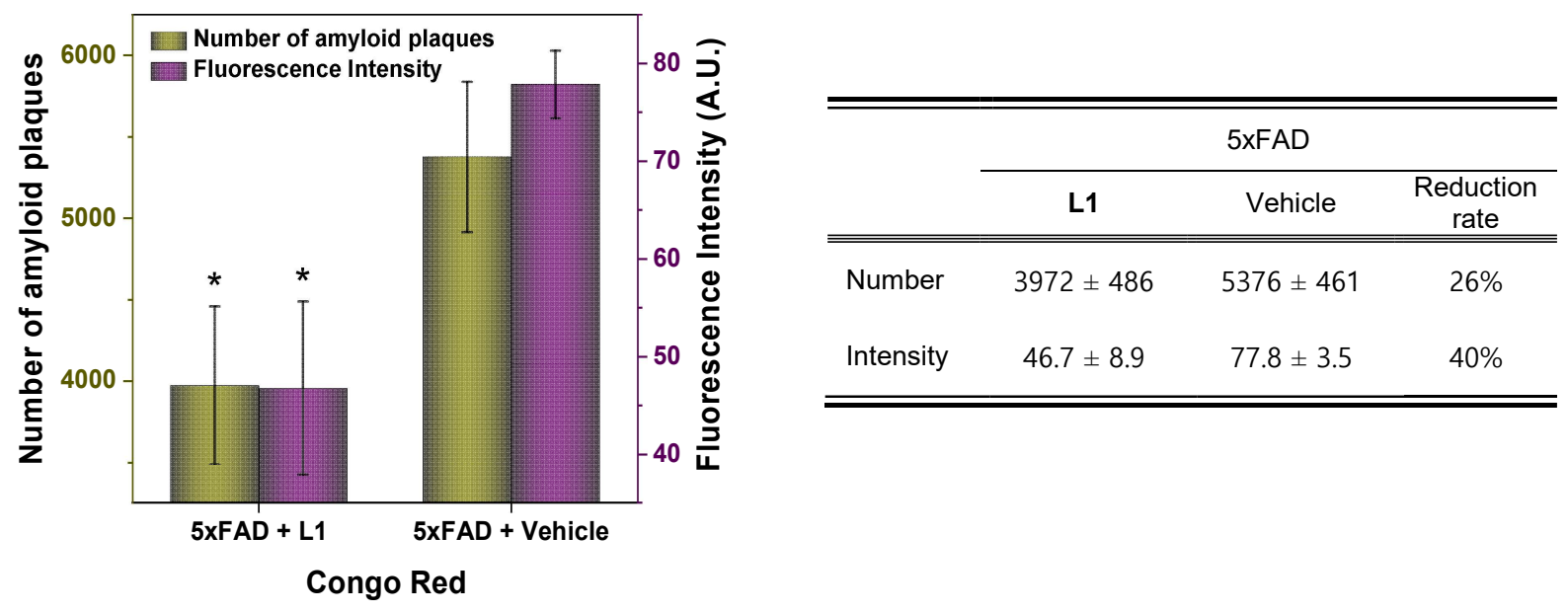

Figure S7. (a) Representative fluorescence microscopy images of the Congo Red-stained brain sections from 5xFAD mouse treated with $\mathrm{L} 1$ or vehicle. The fluorescence of Congo Red was monitored with a standard TRITC filter set. The image in right lane is a magnified view. Scale bar: $1 \mathrm{~mm}$. (b) The total number and fluorescence intensity of amyloid plaques in the Congo Redstained brain sections from $5 \mathrm{xFAD}$ mouse treated with $\mathrm{L} 1$ or vehicle. When compared to vehicletreated mice, the amyloid burden in the L1-treated mice were reduced by $26 \%$ and $40 \%$ based on the number of amyloid plaques and fluorescence intensity, respectively. The fluorescence intensity and number of amyloid plaques were obtained as the sum obtained from ten brain sections per mouse. Error bars are presented as standard deviation $(n=3$ mice $)$, and the statistical analysis was evaluated according to one-way ANOVA $(* \mathrm{p}<0.05)$. 


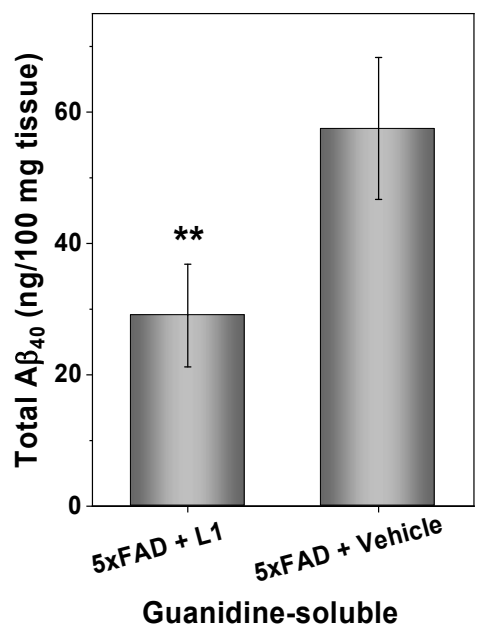

Figure S8. Guanidine-soluble $A \beta_{40}$ levels from brain tissues, quantified using ELISA. Compared to the vehicle-treated mice brains, the total amount of the $A \beta_{40}$ species in the L1-treated mice was reduced by $49 \%$ in the guanidine-soluble brain homogenates. Error bars represent standard deviations ( $\mathrm{n}=3$ mice), and the statistical analysis was evaluated according to one-way ANOVA $(* * p<0.01)$. Note: the amounts of PBS-soluble A $\beta_{40}$ species obtained from the vehicle- and L1treated mice brains were too small to be detected reproducibly by ELISA. 

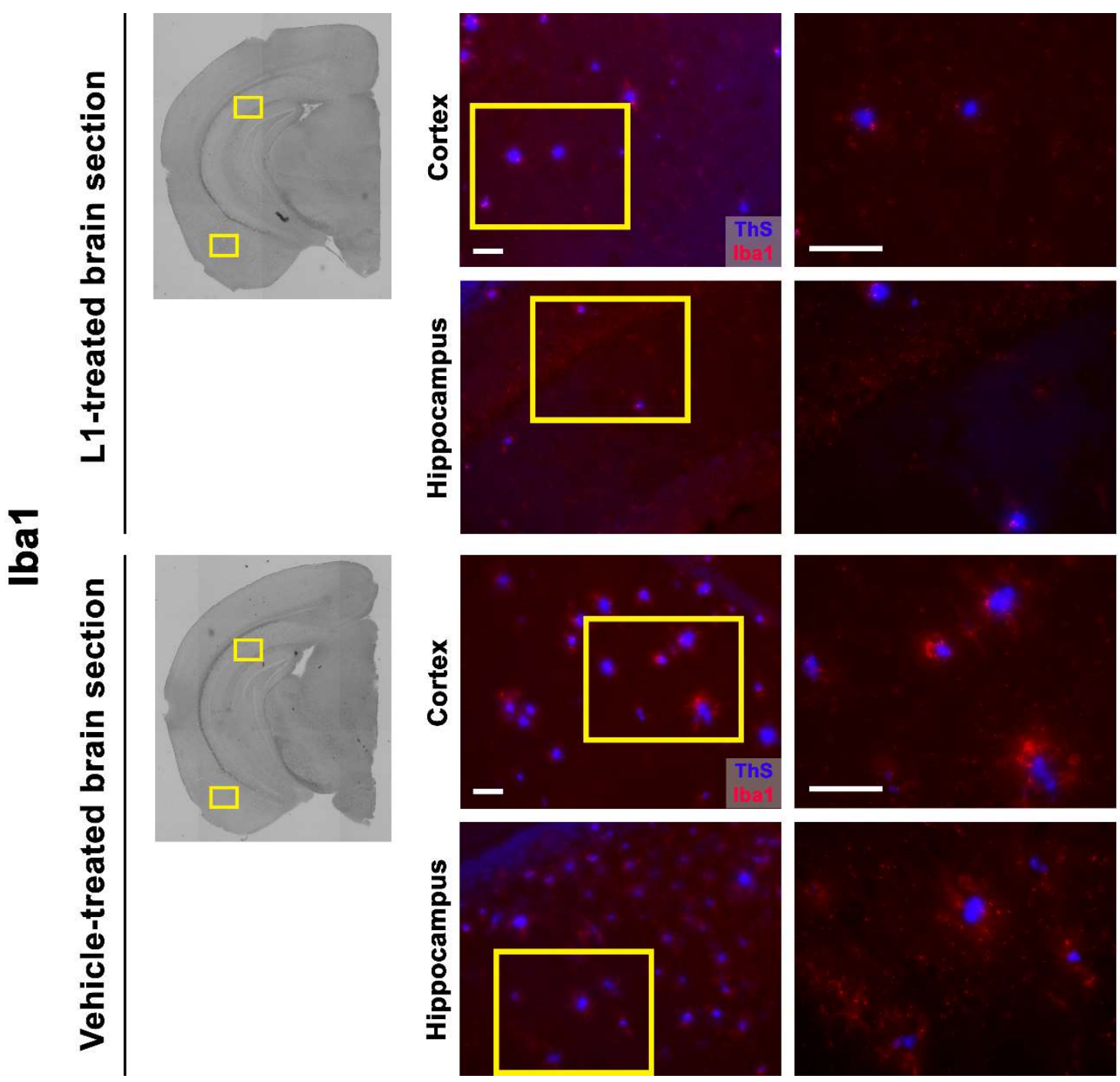

Figure S9. Representative bright field and fluorescence images of the AF594-Iba1-stained brain sections from 5xFAD mice treated with L1 or vehicle. The fluorescence of AF594-Iba1 was monitored with a standard Texas Red filter set. The regions highlighted with a yellow rectangle in the cortex and hippocampus areas of each brain section were magnified and shown in the right panels. All fluorescence images are the maximum intensity projection images obtained from $30 \mathrm{Z}-$ sections collected at $1 \mu \mathrm{m}$ intervals. Color: red, AF594-Iba1 antibody; blue, ThS. Scale bar: 50 $\mu \mathrm{m}$. 

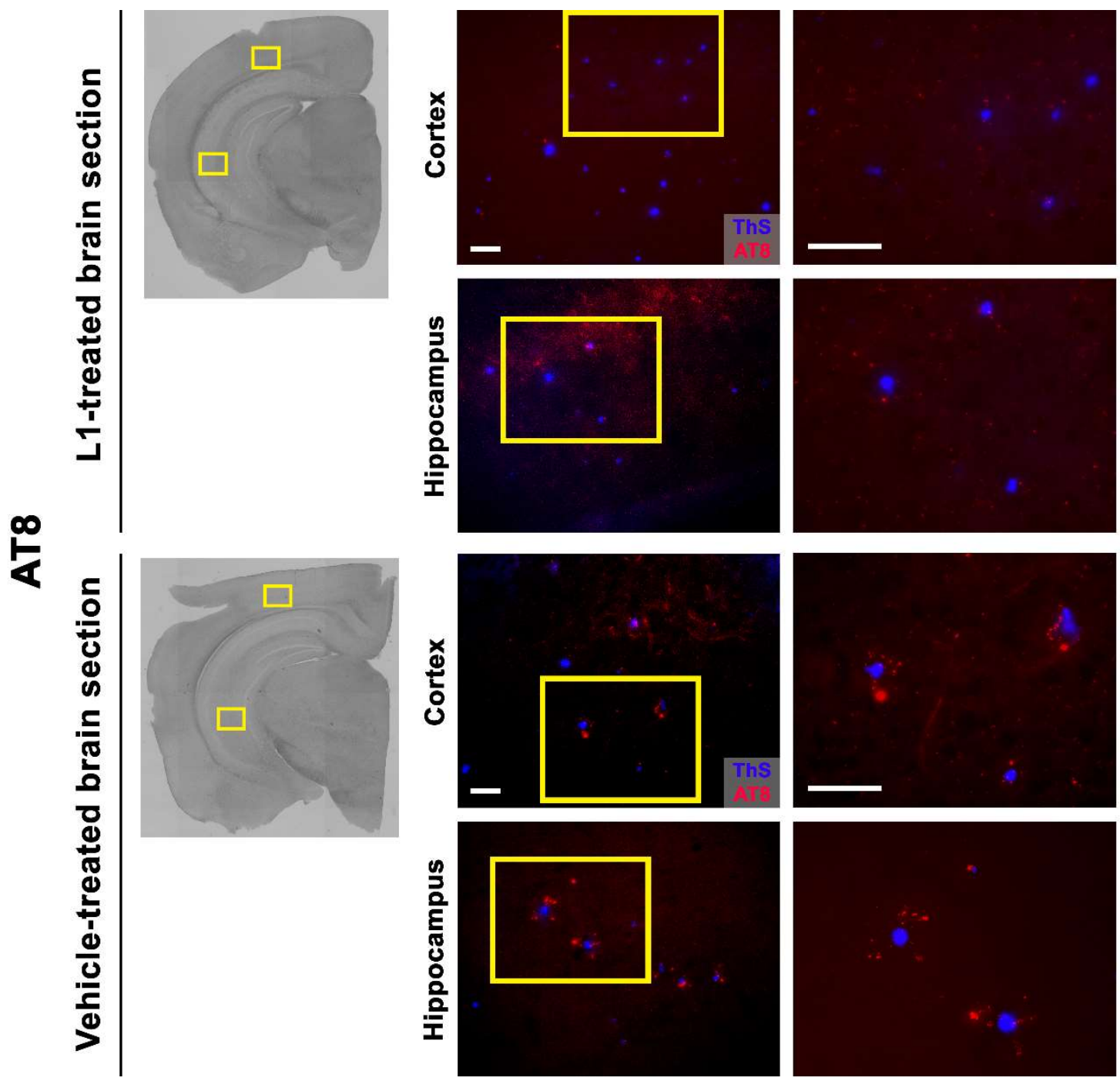

Figure S10. Representative bright field and fluorescence images of the AF594-AT8-stained brain sections from 5xFAD mouse treated with L1 or vehicle. The fluorescence of AF594-AT8 was monitored with a standard Texas Red filter set. The regions highlighted with a yellow rectangle in the cortex and hippocampus areas of each brain section were magnified and shown in the right panels. All fluorescence images are the maximum intensity projection images obtained from $30 \mathrm{Z}$ sections collected at $1 \mu \mathrm{m}$ intervals. Color: red, AF594-AT8 antibody; blue, ThS. Scale bar: 50 $\mu \mathrm{m}$. 


\section{References}

1. Schwetye, K. E.; Cirrito, J. R.; Esparza, T. J.; Mac Donald, C. L.; Holtzman, D. M.; Brody, D. L., Traumatic brain injury reduces soluble extracellular amyloid- $\beta$ in mice: A methodologically novel combined microdialysis-controlled cortical impact study. Neurobiol. Dis. 2010, 40 (3), 555-564.

2. Klein, W. L., A $\beta$ toxicity in Alzheimer's disease: globular oligomers (ADDLs) as new vaccine and drug targets. Neurochem. Int. 2002, 41 (5), 345-352.

3. Re, R.; Pellegrini, N.; Proteggente, A.; Pannala, A.; Yang, M.; Rice-Evans, C., Antioxidant activity applying an improved ABTS radical cation decolorization assay. Free Radic. Biol. Med. 1999, 26 (9), 1231-1237.

4. Jones, M. R.; Mathieu, E.; Dyrager, C.; Faissner, S.; Vaillancourt, Z.; Korshavn, K. J.; Lim, M. H.; Ramamoorthy, A.; Wee Yong, V.; Tsutsui, S.; Stys, P. K.; Storr, T., Multi-targetdirected phenol-triazole ligands as therapeutic agents for Alzheimer's disease. Chem. Sci. 2017, $8(8), 5636-5643$.

5. Zhang, Y.; Rempel, D. L.; Zhang, J.; Sharma, A. K.; Mirica, L. M.; Gross, M. L., Pulsed hydrogen-deuterium exchange mass spectrometry probes conformational changes in amyloid beta (A $\beta$ ) peptide aggregation. Proc. Natl. Acad. Sci. U.S.A. 2013, 110, 14604-14609.

6. Morris, A. M.; Watzky, M. A.; Agar, J. N.; Finke, R. G., Fitting neurological protein aggregation kinetic data via a 2-step, minimal/"Ockham's razor" model: the Finke-Watzky mechanism of nucleation followed by autocatalytic surface growth. Biochemistry 2008, 47 (8), 2413-2427.

7. Yan, P.; Bero, A. W.; Cirrito, J. R.; Xiao, Q.; Hu, X.; Wang, Y.; Gonzales, E.; Holtzman, D. M.; Lee, J.-M., Characterizing the appearance and growth of amyloid plaques in APP/PS1 mice. J. Neurosci. 2009, 29 (34), 10706-10714. 